\title{
DESEMPENHO DE PAINÉIS OSB COM ADESIVOS COMERCIAIS E TÂNICO DE BARBATIMÃO ${ }^{1}$
}

\begin{abstract}
Amelia Guimarães Carvalho², Marina Rates Pires ${ }^{3}$, Antonio José Vinha Zanuncio ${ }^{2}$, Rafael Farinassi Mendes $^{4}$, Fábio Akira Mori ${ }^{5}$ e Lourival Marin Mendes ${ }^{5}$

RESUMO - O objetivo deste trabalho foi avaliar as propriedades físicas e mecânicas de painéis OSB produzidos com adesivos comerciais e tânico de Stryphnodendron adstringens (Mart.) Coville. Os adesivos comerciais ureia formaldeído (UF), fenol formaldeído (FF) e os taninos da Acacia mearnsi foram obtidos por doação por respectivas empresas produtoras, enquanto que o tanino de barbatimão foi obtido em laboratório. Todos adesivos tiveram as propriedades dos adesivos determinadas. Foram produzidos painéis OSB com os adesivos ureia-formaldeído, fenol-formaldeído, tanino-formaldeído a partir da acácia e do barbatimão. Os painéis foram produzidos com partículas do tipo strand, geradas a partir da madeira de pinus, com teor de adesivo de $8 \%$ a uma pressão de

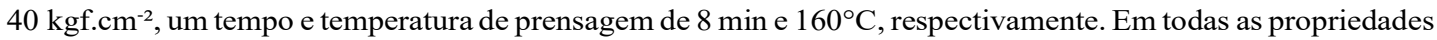
avaliadas, o desempenho dos painéis produzidos com adesivo tânico de barbatimão foi melhor quando comparado com o dos painéis produzidos com o adesivo tânico de acácia. Os painéis produzidos com os adesivos UF, FF e tânico de barbatimão atenderam a todos os prerrequisitos estipulados pela norma de comercialização. Tal fato demonstra grande potencial de utilização dos taninos de barbatimão para produção de painéis OSB.
\end{abstract}

Palavras-chave: Taninos; Adesivos naturais; Oriented strand board.

\section{OSB PANELS PERFORMANCE WITH COMMERCIAL ADHESIVES AND TANNIC ADHESIVE FROM BARBATIMÃO}

\begin{abstract}
The aim of this study was to evaluate the physical and mechanical properties of OSB (Oriented Strand Board) panels produced with commercial adhesives and tannic adhesive from Stryphnodendron adstringens (Mart.) Coville. The commercial adhesives, urea formaldehyde (UF), phenol formaldehyde (FF) and Acacia mearnsi tannin were obtained by donation by respective manufacturing companies, while the tannins of barbatimao were obtained in the laboratory. The adhesives had the adhesives properties determined. OSB panels were produced, with the adhesives urea-formaldehyde, phenol-formaldehyde, tannin-formaldehyde from Acacia and Stryphnodendron. The panels were produced with strand particles generated from pine wood with an adhesive content of $8 \%$ at a $40 \mathrm{kgf.cm} \mathrm{cm}^{-2}$ of pressure, a time and pressing temperature of 8 minutes and $160^{\circ} \mathrm{C}$, respectively. In all of the properties evaluated, the performance of the panels made with tannic adhesive from barbatimão was better when compared to panels manufactured with the tannic adhesive from acacia. The panels produced with urea-formaldehyde, phenol-formaldehyde and tannic adhesive of barbatimão met all requirements stipulated by the marketing standard. Fact that shows great use potential for tannin of barbatimão for OSB panels production.
\end{abstract}

Keywords: Tannins; Natural adhesives; Oriented strand board.

\footnotetext{
${ }^{1}$ Recebido em 06.08.2013 aceito para publicação em 21.10.2015.

${ }^{2}$ Universidade Federal de Viçosa, Programa de Pós-graduação em Ciência Florestal, Viçosa, MG - Brasil. E-mail: $<$ ameliagcarvalho@gmail.com>e < ajvzanuncio@yahoo.com.br>.

${ }^{3}$ Universidade Federal de Lavras, Graduanda em Engenharia Florestal, Lavras, MG - Brasil. E-mail: <marinarates@hotmail.com>.

${ }^{4}$ Universidade Federal de Lavras, Departamento de Engenharia, Lavras, MG - Brasil. E-mail: <rafaelfarinasi@gmail.com>.

${ }^{5}$ Universidade Federal de Lavras, Departamento de Ciências Florestais, Lavras, MG - Brasil. E-mail: <morif@dcf.ufla.br $>$ e $<$ lourival@dcf.ufla.br $>$.
} 


\section{INTRODUÇÃO}

O painel do tipo Oriented Strand Board (OSB) é formado por partículas retangulares tipo "strand" dispostas em três camadas parcialmente cruzadas, o que confere melhor distribuição da resistência nas direções longitudinal e transversal, além de melhorar a estabilidade dimensional do painel.

O painel OSB é importante em aplicações decorativas e estruturais, competindo com os compensados. Suas propriedades mecânicas dependem da qualidade das ligações entre as partículas de madeira, o que é determinado pela quantidade e tipo de adesivo (DUNKY; PIZZI, 2002).

Os adesivos sintéticos consistem em oligômeros ou polímeros que são sintetizados a partir de derivados do petróleo. A ideia de substituir materiais de fontes não renováveis por fontes renováveis, aliada aos instáveis preços dos recursos petrolíferos, incentivam a busca por fontes renováveis que gerem produtos com qualidade igual ou superior à do material não renovável.

Os taninos são compostos fenólicos potenciais para a produção de adesivos substitutos dos sintéticos utilizados em produtos de madeira reconstituída (TROSA; PIZZI, 2001; BALLERINI et al., 2005; PIZZI, 2006; CARNEIRO, et al., 2009; MOUBARIK et al., 2009; PING et al., 2011; CARVALHO et al., 2014). São classificados como metabólitos secundários, importantes no mecanismo de defesa do vegetal contra a radiação solar, herbivoria e organismos patogênicos. Além da utilização como adesivo de madeira, os taninos são amplamente utilizados no curtimento de peles, no tratamento de água potável (floculante), agente antioxidante em águas e caldeiras, desenvolvimento de sabor adstringente na fabricação de bebidas, na produção de produtos farmacêuticos (PIZZI, 1994) e na indústria sucroalcooleira para clarificação de açúcar.

Entre as espécies do Cerrado que apresentam potencial tanífero, o Stryphnodendron adstringens (Mart.) Coville, conhecido popularmente como barbatimão, tem obtido destaque como principal uso de valor econômico, tal a extração de taninos encontrados em suas cascas e folhas. As folhas apresentam em torno de $27 \%$ a $32 \%$ (base massa de matéria seca de material de tanino) nos meses de maio e setembro (ALMEIDA et al., 2008), enquanto o rendimento médio em taninos condensados da casca de barbatimão é de $40 \% \mathrm{em}$ relação à massa de matéria seca do material (MORI et al., 2006).

Com base no exposto, este trabalho teve por objetivo avaliar as propriedades físicas e mecânicas de painéis OSB produzidos com adesivos comerciais e adesivos tânicos de Stryphnodendron adstringens (Mart.) Coville.

\section{MATERIAL E MÉTODOS}

\subsection{Obtenção dos adesivos}

Os adesivos comerciais ureia formaldeído (UF), fenol formaldeído (FF) e os taninos da Acacia mearnsi foram obtidos por doação por respectivas empresas produtoras.

Para a produção do adesivo tânico de barbatimão, foi realizada a coleta das cascas em um fragmento de Cerrado, localizado no Município de Lavras, Minas Gerais. As cascas coletadas foram misturadas, fragmentadas e, na sequência, secas ao ar livre. Uma vez secas, as cascas foram moídas em moinho martelo, com a utilização de uma peneira com $2 \mathrm{~mm}^{2}$ de abertura.

A extração dos polifenóis foi realizada de acordo com o recomendado por Mori et al. (2003) para o Stryphnodendron adstringens (Mart.) Coville. Em seguida, o material foi filtrado, empregando-se uma peneira de malha de $1 \mathrm{~mm}^{2}$ e uma bomba de vácuo, em cadinhos de vidro sinterizado de porosidade 2 . Os extratos foram distribuídos em bandejas de vidro e levados à estufa, a uma temperatura de $40^{\circ} \mathrm{C}$ até a secagem total. Por fim, foram moídos manualmente com a utilização de um graal de porcelana com pistilo.

Os adesivos termofixos à base de taninos (barbatimão e de acácia) foram sintetizados misturando-se os taninos em pó com água, em agitador com uma rotação de 3000 RPM e em uma concentração aproximada de 50\% de sólidos totais. Após $24 \mathrm{~h}$ de hidratação, foram adicionados $8 \%$ do paraformaldeído (agente ligante) em relação à massa de matéria seca de taninos em pó.

\subsection{Propriedades dos adesivos}

A viscosidade foi determinada utilizando um viscosímetro do tipo Copo Ford (Universal) e seguindo os parâmetros estipulados pela Norma ASTM D-1200 (ASTM, 1994).

O tempo de gel foi obtido utilizando amostras de 5 g de adesivo de cada tratamento, colocadas em tubos 
de ensaio de $15 \mathrm{~cm}$ de altura e $2 \mathrm{~cm}$ de diâmetro. $\mathrm{O}$ conjunto tubo-bastão foi mergulhado em banho de glicerina $130^{\circ} \mathrm{C}$. Com o auxílio do bastão, o líquido foi agitado com movimento vertical, em razão do aumento da resistência do adesivo ao atingir a "fase de gel". O tempo de gelatinização ( gel time) de cada tratamento foi entre a imersão do tubo na glicerina e o endurecimento da amostra.

A determinação do teor de sólidos foi realizada pesando $1 \mathrm{~g}$ do adesivo e secando-o na estufa a 103 $\pm 3^{\circ} \mathrm{C}$ até peso constante. O percentual do teor de sólidos foi calculado pela divisão entre a massa final pela massa inicial vezes 100 .

$\mathrm{O} \mathrm{pH}$ dos extratos aquosos foram determinados pelo pH-metro, após 4 min de contato com o adesivo.

\section{2..3. Produção dos painéis OSB}

Os painéis OSB foram produzidos com madeira de Pinus oocarpa, com 30 anos, e quatro diferentes adesivos: ureia formaldeído (UF), fenol formaldeído (FF), adesivo tânico de acácia-negra e adesivo tânico de barbatimão, sendo cada adesivo um tratamento. Para cada tratamento foram produzidos três painéis, com dimensões de 48 x 48 x 1,5 cm (comprimento, largura e espessura, respectivamente) e densidade nominal de 0,65 g. $\mathrm{cm}^{-3}$.

As árvores derrubadas foram seccionadas em discos e toras. Os discos foram utilizados para determinação da densidade básica da madeira, conforme a Norma NBR 11941 (2003). Enquanto as toras foram desdobradas, mediante a utilização de serra de fita, em tábuas de $30 \mathrm{~mm}$ de espessura.

As tábuas foram processadas em desengrossadeira para ajustar as suas espessuras em $25 \mathrm{~mm}$. Posteriormente, estas foram, então, seccionadas com uma serra circular em blocos com $90 \mathrm{~mm}$ de largura, na direção das fibras. Em seguida, os blocos foram submersos em água até a completa saturação. Uma vez saturados, esses foram processados em um picador de disco, com as facas ajustadas para gerarem partículas com espessuras em torno de $0,60 \mathrm{~mm}$, obtendo, assim, partículas tipo strand com as dimensões de $25 \times 90$ x 0,60 mm (largura x comprimento x espessura, respectivamente)

As partículas após a secagem, em estufa com circulação forçada de ar até a umidade de 3\% (base massa de matéria seca das partículas), foram encaminhadas para uma encoladeira do tipo tambor giratório, onde se realizou a aplicação de $8 \%$ de adesivo (base massa de matéria seca das partículas), conforme tratamentos preestabelecidos.

$\mathrm{Na}$ formação do colchão, as partículas foram dispostas em três camadas, cujas proporções, em massa, foram de 25:50:25 (face, miolo e face, respectivamente), sendo a camada do miolo orientada de forma perpendicular às camadas da face.

Depois de formado o colchão de partículas, este foi, então, levado para uma prensa hidráulica manual, para efetuar a sua pré-prensagem a frio, o que ocorreu na pressão de $4 \mathrm{kgf.cm}{ }^{-2}$. Posteriormente, o colchão foi levado para a prensagem a quente, com separadores de $15 \mathrm{~mm}$ de espessura, onde passaram por um ciclo de prensagem de $8 \mathrm{~min}, 160^{\circ} \mathrm{C}$ e $40 \mathrm{kgf} . \mathrm{cm}^{-2}$.

\subsection{Avaliação das propriedades físico-mecânicas}

As dimensões dos corpos de prova, os procedimentos para realização dos testes de absorção de água (AA) e o inchamento em espessura (IE), após duas e $24 \mathrm{~h}$ de imersão em água, taxa de não retorno em espessura (TNRE), densidade aparente e ligação interna, foram definidos com base na Norma americana ASTM D 1037 (2006). Para determinação do módulo de ruptura (MOR) e do módulo de elasticidade (MOE) à flexão estática, nas direções paralela e perpendicular foi empregada a Norma DIN 52362 (1982).

\subsection{Análise estatística}

Foi considerado um delineamento inteiramente casualizado (DIC). Para a diferenciação entre os tratamentos, foi realizada a análise de variância a $5 \%$ de significância, seguida do teste de média de Tukey também a 5\% de significância.

De forma preliminar às análises de variância, foi realizado o teste de homogeneidade de variâncias (teste de Bartlett a 5\% de significância) e de normalidade (teste de Shapiro-Wilk a 5\% de significância).

\section{RESULTADOS}

\subsection{Propriedades dos adesivos}

Os adesivos UF, FF e à base de taninos de barbatimão e de acácia apresentaram viscosidade de 150, 218, 459 e $430 \mathrm{cP}$.

Revista Árvore, Viçosa-MG, v.39, n.6, p.1155-1163, 2015 
Os adesivos tânicos apresentaram tempo de gel de $56 \mathrm{seg}$ para o barbatimão e de $103 \mathrm{seg}$ para o de acácia. Os adesivos UF e FF exibiram tempo de gel de 120 e 279 seg, respectivamente.

O teor de sólidos dos adesivos tânicos ficou próximo de $50 \%$, valor esse estipulado para a sua confecção. Os adesivos UF e FF apresentaram valores de 46,8 e 53,8\%, superiores aos tânicos na ordem de 8,9 e 13, respectivamente, enquanto os tânicos, valores inferiores de $\mathrm{pH}$ na ordem de 4,9 e 5,3, para os adesivos de barbatimão e de acácia, respectivamente.

\subsection{Densidade aparente e razão de compactação dos painéis}

A densidade básica da madeira de Pinus oocarpa foi de $0,42 \mathrm{~g} \cdot \mathrm{cm}^{-3}$. Não houve diferença estatística entre as densidades básicas aparentes nos diferentes tratamentos, com valores de 0,$54 ; 0,54 ; 0,56 ;$ e $0,57 \mathrm{~g} . \mathrm{cm}^{-3}$, para os painéis produzidos com os adesivos UF (T1), FF (T2), adesivo tânico de barbatimão (T3) e tânico de acácia (T4), respectivamente. Com isso, a razão de compactação de T1, T2 e T3 foi de 1,3 e a de T4 foi de 1,4 .

\subsection{Absorção de água}

Os paineís produzidos com o adesivo UF, FF e adesivo tânico de barbatimão apresentaram valores médios próximos, variando de 72,3 a $79,6 \%$ para AA $2 \mathrm{~h}$ e de 84,8 a $89,3 \%$ para AA $24 \mathrm{~h}$. Apenas os painéis produzidos com o adesivo tânico de acácia diferiram estatisticamente dos demais, apresentando valores de absorção de água superiores (Figura 1).

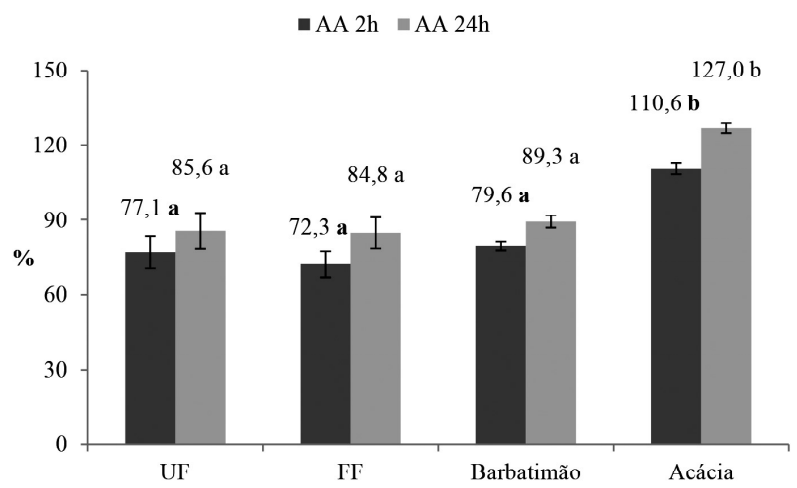

Figura 1 - Absorção de água dos painéis OSB após 2 e 24 $\mathrm{h}$ de imersão em água.

Figure 1 - Water absorption of the OSB panels after 2 and 24 hours of immersion in water.

\subsection{Inchamento em espessura e taxa de não retorno em espessura}

Assim, como na absorção de água, os painéis produzidos com o adesivo UF, FF e adesivo tânico de barbatimão apresentaram valores médios próximos e não diferiram estatisticamente entre si. Valores variando de 8 a $12,3 \%$ para IE 2 h; de 10 a $14,3 \%$ para IE $24 \mathrm{~h}$ e de 7,3 a $10,2 \%$ para TNRE. Apenas os paineís produzidos com o adesivo tânico de acácia diferiu estatisticamente dos demais, apresentando valores de IE 2 h (26\%), IE $24 \mathrm{~h}(28,7 \%)$ e TNRE (25,3\%) superiores (Figura 2).

\subsection{Ligação interna}

Os paineís produzidos com os adesivos UF e tânico de barbatimão não diferiram estatisticamente, apresentando valores intermediários em relação ao FF, que obteve maiores valores. O adesivo tânico de acácia obteve os menores valores de ligação interna (Figura 3).

\subsection{Módulo de ruptura e de elasticidade à flexão estática}

O módulo de ruptura perpendicular $(M O R \perp)$ não diferiu entre os tratamentos. O módulo de ruptura paralelo (MOR//) foi superior nos tratamentos com os adesivos UF e FF, seguido pelo tânico barbatimão e, por fim, o tânico de acácia (Figura 4).

O módulo de elasticidade paralelo (MOE //) não diferiu entre os tratamentos. Para módulo de elasticidade perpendicular $(\mathrm{MOE} \perp)$, o adesivo de acácia apresentou

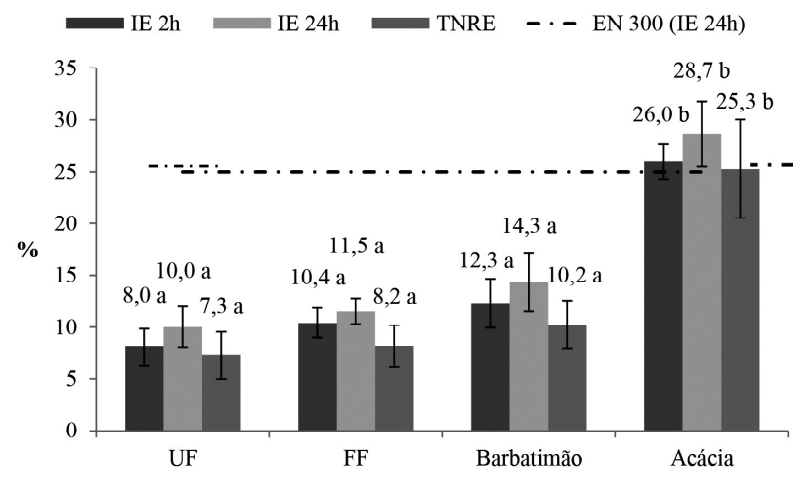

Figura 2 - Inchamento em espessura dos painéis OSB após 2 e $24 \mathrm{~h}$ de imersão em água e a taxa de não retorno em espessura.

Figure 2-Swelling in thickness of the OSB panels after 2 and 24 hours of immersion in water and the nonreturn rate in thickness. 
o menor valor médio, diferindo estatisticamente do tratamento com o adesivo FF, que apresentou o maior valor médio. Os tratamentos com o adesivo UF e o tânico de barbatimão foram estatisticamente iguais a todos os tratamentos (Figura 5).

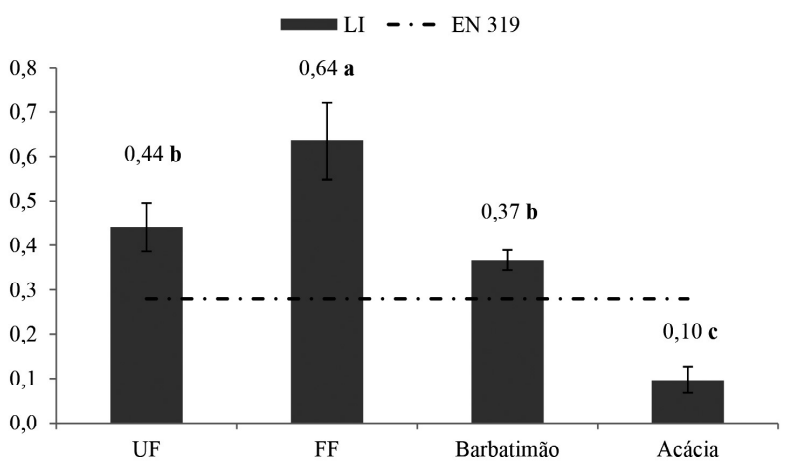

Figura 3 - Ligação interna dos painéis OSB.

Figure 3 - Internal bond of the OSB panels.

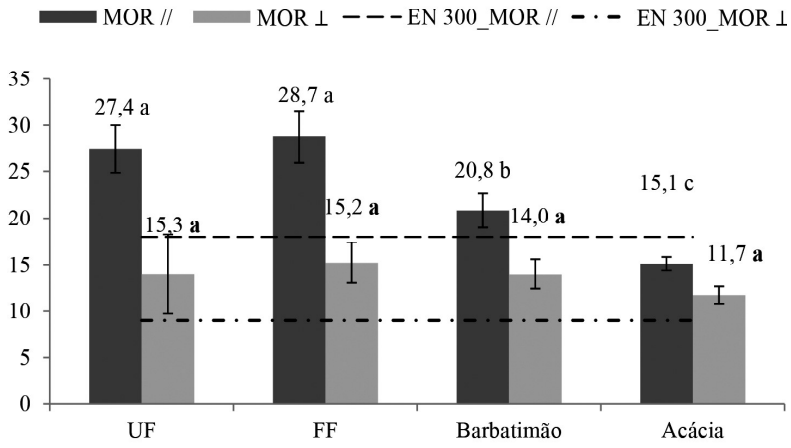

Figura 4 - Valores médios dos módulos de ruptura dos painéis OSB.

Figure 4-Mean values of rupture modulus of the OSB panels.

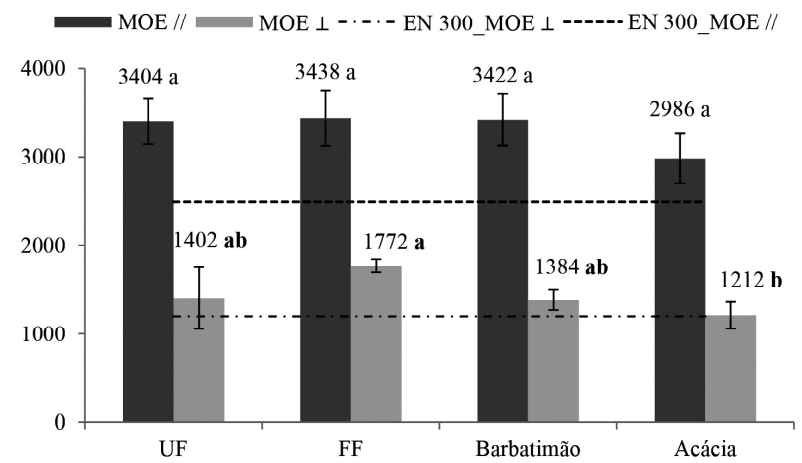

Figura 5 - Valores médios dos módulos de elasticidade dos painéis OSB.

Figure 5-Mean values of elasticity modules of the OSB panels.

\section{DISCUSSÃO}

\subsection{Propriedades dos adesivos}

Adesivos tânicos apresentaram maior viscosidade em comparação com os adesivos fenolformaldeído (FF) e ureia-formaldeído (UF). Segundo Iwakiri (2005), o valor de viscosidade máximo para aplicação do adesivo em painéis particulados é de até $1.000 \mathrm{cP}$. Assim, apesar de os adesivos tânicos apresentarem viscosidades superiores, eles estão em padrões aceitáveis para aplicação, por aspersão, na produção de painéis particulados.

O baixo tempo de gel do adesivo tânico evidencia a alta reatividade dos taninos em relação ao paraformaldeído, o que promove a cura mais rápida do adesivo e diminui sua vida útil.

O teor de sólidos indica a porcentagem de sítios reativos com o agente ligante. Assim, maior teor de sólidos do adesivo torna resistente a sua linha de cola, porém se deve levar em consideração que teores de sólido muito altos também prejudicam a aplicação do adesivo via aspersão, devido ao aumento da sua viscosidade.

É possível verificar o carater ácido do adesivo tânico e básico dos adesivos fenolformaldeído e ureiaformaldeído, com base no $\mathrm{pH}$.

Teodoro e Lelis (2003), trabalhando com a casca de Eucalyptus pellita, encontraram valores médios de 3,9 para o $\mathrm{pH}, 45,9 \%$ para o teor de sólidos, $62 \mathrm{seg}$ para o tempo de gel e de $826,5 \mathrm{cP}$ para a viscosidade. Enquanto Goulart et al. (2012), avaliando as propriedades de adesivo tânico da casca e da folha de barbatimão, encontraram valores médios para o adesivo da casca de barbatimão de 4,9 para o $\mathrm{pH}, 54,2 \%$ para o teor de sólidos, $1488 \mathrm{cP}$ para a viscosidade e $42 \mathrm{seg}$ para o tempo de gel.

Os valores médios de viscosidade com os taninos de barbatimão e de acácia foram inferiores aos encontrados na literatura, estando as outras propriedades de acordo com os encontrados na literatura.

\subsection{Densidade aparente e razão de compactação}

De acordo com a norma comercial americana para painéis particulados, CS 236-66 (COMMERCIAL STANDARD, 1968), todos os painéis deste trabalho se enquadram na densidade C (abaixo de 0,600 g. $\mathrm{cm}^{-3}$ ).

Revista Árvore, Viçosa-MG, v.39, n.6, p.1155-1163, 2015 
Segundo Maloney (1993), a razão de compactação ideal de painéis particulados é de 1,3 a 1,6, promovendo, assim, adequada compactação das partículas e, consequentemente, boas propriedades físicas e mecânicas dos painéis. Portanto, todos os tratamentos estão dentro da faixa adequada de razão de compactação citada.

\subsection{Absorção de água}

A absorção de água do tratamento com o adesivo tânico de barbatimão foi inferior ao que utilizou adesivo de acácia e igual aos painéis produzidos com os adesivos FF e UF.

Vital et al. (2004) produziram painéis de flocos utilizando a madeira de Pinus elliottii, densidade aparente média de 0,56 g.cm ${ }^{-3}$ e com adesivos tânicos de Eucalyptus grandis e Eucalyptus pellita a um teor de $8 \%$ de adesivo (base de partículas secas). A AA2h e $24 \mathrm{~h}$ dos painéis produzidos com adesivo de $E$. grandis foram de 68,7 e $85 \%$, respectivamente, e com o adesivo de E. pellita de 88 e $108,9 \%$, respectivamente.

Salari et al. (2013), ao avaliarem a qualidade de painéis OSB produzidos com $10 \%$ de adesivo ureiaformaldeído e com densidade aparente de $0,70 \mathrm{~g} . \mathrm{cm}^{-3}$, obtiveram valores médios de 66,1 e $120,9 \%$ para AA2h e AA24h, respectivamente.

Os dados encontrados são compatíveis com os da literatura. Quando comparados apenas os dois adesivos tânicos, observou-se melhor desempenho do adesivo de barbatimão perante do de acácia.

\subsection{Inchamento em espessura}

A norma europeia de comercialização EN 300 (EUROPEANCOMMITTEEFOR STANDARDIZATION, 2006) estipula $25 \%$ como valor máximo de inchamento em espessura após 24 h de imersão em água, para painéis OSB do tipo 1, utilizados em ambiente sem contato com a umidade. Assim, os painéis com adesivos FF, UF e tânico de barbatimão atenderam à Norma. Apenas o painel produzido com o adesivo tânico de acácia apresentou valor de IE24h superior, de 28,7\%.

Vital et al. (2004) produziram painéis de flocos utilizando a madeira de Pinus elliottii com adesivos tânicos de taninos de Eucalyptus grandis e Eucalyptus pellita. O inchamento em espessura após 2 e $24 \mathrm{~h}$ de imersão em água dos painéis com adesivos tânicos de E. grandis foi de 23,7 e $38,4 \%$, respectivamente, enquanto para os painéis com o adesivo tânicos de E. pellita os valores médios foram de 36,1 e 57,4\%, respectivamente.

Os painéis OSB produzidos por Okino et al. (2007) com partículas geradas a partir da madeira de cypress (Cupressus glauca Lam.), com adesivo ureia-formaldeído a um teor de $8 \%$ (base massa de matéria seca das partículas), obtiveram inchamento em espessura após 2 e 24 h de imersão em água de 22,7 e 30,9\%, respectivamente.

Salari et al. (2013), ao avaliarem a qualidade de painéis OSB produzidos com $10 \%$ de adesivo ureiaformaldeído e com densidade aparente de $0,70 \mathrm{~g} . \mathrm{cm}^{-3}$, obtiveram valores médios de 43,2 e $93,5 \%$ para o IE2h e IE24h, respectivamente.

Os valores tanto de IE $2 \mathrm{~h}$ quanto IE $24 \mathrm{~h}$ dos tratamentos utilizando os adesivos UF, FF e tânico de barbatimão foram inferiores aos obtidos na literatura. Já em relação aos painéis produzidos com o adesivo de acácia, apenas para o IE $24 \mathrm{~h}$ foram obtidos valores inferiores.

\subsection{Ligação interna}

A melhor ligação interna foi obtida para os painéis produzidos com o adesivo fenolformaldeído, os adesivos UF e tânico de barbatimão proporcionaram valores inferiores, porém superiores aos do adesivo de acácia.

Sellers e Miller (2004), ao estudarem a qualidade de painéis OSB produzidos com $10 \%$ de adesivos tânicos de acácia, quebracho e Pinus radiata e densidade aparente de 0,56 a 0,70 g. $\mathrm{cm}^{-3}$, obtiveram valores de ligação interna entre 0,16 e 0,35 $\mathrm{MPa}$.

Salari et al. (2013), ao avaliarem a qualidade de painéis OSB produzidos com $10 \%$ de adesivo ureiaformaldeído e com densidade aparente de $0,70 \mathrm{~g} . \mathrm{cm}^{-3}$, obtiveram valores médios de ligação interna de 0,45 MPa.

Os painéis com adesivo de tanino de barbatimão, FF e UF apresentaram valores coerentes com os encontrados na literatura e atenderam ao mínimo requerido pela Norma EN 300 (2006), que estipula o valor mínimo de $0,30 \mathrm{MPa}$ para a propriedade ligação interna em painéis OSB do tipo 1. Apenas os painéis produzidos com o adesivo de acácia não atenderam à exigência dessa Norma. 


\subsection{Módulo de ruptura e de elasticidade à flexão estática}

Sellers e Miller (2004), ao estudarem a qualidade de painéis OSB produzidos com 10\% de adesivos tânicos de acácia, quebracho e pinus radiata e densidade aparente de 0,56 a 0,70 g. $\mathrm{cm}^{-3}$, obtiveram valores médios entre 18,5 e 26,9 MPa para o MOR paralelo e entre 2.754 a 3.802 MPa para o MOE paralelo.

Okino et al. (2007) produziram painéis OSB com a madeira de cypress (Cupressus glauca Lam.), a uma densidade nominal de 0,7 g. $\mathrm{cm}^{-3}$ e $8 \%$ de adesivo ureiaformaldeído e encontraram valores de $\mathrm{MOE} / / \mathrm{e} \perp$ na ordem de 4.667 e $2.524 \mathrm{MPa}$ e para MOR // e $\perp$ na ordem de 50,9 e 34,6 $\mathrm{MPa}$, respectivamente.

Salari et al. (2013), ao avaliarem a qualidade de painéis OSB produzidos com $10 \%$ de adesivo ureiaformaldeído e com densidade aparente de $0,70 \mathrm{~g} . \mathrm{cm}^{-3}$, obtiveram valores médios de MOR e MOE paralelo de 19,3 e $2.385 \mathrm{MPa}$, respectivamente.

A Norma EN 300 (2006) estipula o valor mínimo, para painéis OSB do tipo 1 , de 18 e $9 \mathrm{MPa}$ para o MOR paralelo e perpendicular e de 2.500 e $1.200 \mathrm{MPa}$ para o MOE paralelo e perpendicular, respectivamente. Assim, todos os tramentos obtiveram seus valores médios superiores ao mínimo estipulado pela Norma citada e apresentaram valores coerentes com os encontrados na literatura.

\section{CONCLUSÃO}

Em todas as propriedades avaliadas, os resultados apresentados pelos painéis produzidos com adesivo tânico de barbatimão foram superiores aos dos painéis produzidos com o adesivo tânico de acácia.

O adesivo de barbatimão foi bastante promissor, pois seus painéis mostraram propriedades estatisticamente iguais às dos painéis produzidos com UF e FF, para as propriedades de absorção de água e inchamento em espessura, ambos após 2 e $24 \mathrm{~h}$ de imerção em água, assim como para a taxa de não retorno em espessura, módulo de elasticidade paralelo e perpendicular e módulo de ruptura perpendicular. Apenas as propriedades de ligação interna e módulo de ruptura paralelo não se mostraram estatisticamente iguais, contudo, mesmo assim, os painéis produzidos com o adesivo tânico de barbatimão atenderam ao mínimo requerido pelas normas europeias.

\section{AGRADECIMENTOS}

À Fundação de Amparo à Pesquisa do Estado de Minas Gerais (FAPEMIG), à Coordenação de Aperfeiçoamento de Pessoal de Nível Superior (CAPES) e ao Conselho Nacional de Desenvolvimento Científico e Tecnológico (CNPq), pelo apoio financeiro.

\section{REFERÊNCIAS}

DE ALMEIDA, N. F.; MORI, F. A.; GOULART, S. L.; MENDES, L. M.; RIBEIRO, A. O. Rendimentos em taninos das folhas de barbatimão [Stryphnodendron adstringens] em diferentes períodos de coleta visando a produção de adesivo para a madeira. In: ENCONTRO BRASILEIRO EM MADEIRA E ESTRUTURAS DE MADEIRA, 11., 2008, Londrina. Anais... Londrina: Universidade Estadual de Londrina, 2008. p.5-8.

\section{ASSOCIAÇÃO BRASILEIRA DE NORMAS} TÉCNICAS - ABNT. CB-29: NBR 11941: Madeira - determinação da densidade básica. Rio de Janeiro: 2003.

\section{AMERICAN SOCIETY FOR TESTING AND MATERIALS - ASTM. ASTM D-1037: standard methods of evaluating properties of wood-base fiber and particles materials. Philadelphia: 2006.}

\section{AMERICAN SOCIETY FOR TESTING AND} MATERIALS - ASTM. ASTM D 1200: Test method for Viscosity by Ford Viscosity. Philadelphia: 1994.

BALLERINI, A., DESPRES, A., PIZZI, A. Nontoxic, zero emission tannin-glyoxal adhesives for wood panels. Holz als Roh- und Werkstoff, v.63, p.477-478, 2005.

CARNEIRO, A. C. O.; VITAL, B. R.; FREDERICO, P. G. U.; CARVALHO, A. M. M. L.; VIDAURRE, G. B. Properties of particleboards glued with adhesives of "angico-vermelho" tannin (Anadenanthera peregrina) and ureaformaldehyde. Revista Árvore, v.33, n.3, p.521-531, 2009.

CARVALHO, A. G.; MORI, F. A.; MENDES, R. F. ZANUNCIO, A. J. V.; DA SILVA, M. G.; MENDES,

Revista Árvore, Viçosa-MG, v.39, n.6, p.1155-1163, 2015 
L. M.; MORI, C. L. S. O. Use of tannin adhesive from Stryphnodendron adstringens (Mart.) Coville in the production of OSB panels.

European Journal of Wood and Wood Products, v.72, n.4, p.425-432, 2014.

COMMERCIAL STANDARD. Mat formed wood particleboard: CS 236-66. [S. 1.: s. n.], 1968.

NORMEN FÜR HOLZFASERPLATTEN SPANPLATTEN SPERRHOLZ - DIN. DIN 52362. Testing of wood chipboards bending test, determination of bending strength: Berlin: 1982. 40p.

DUNKY, M., PIZZI, A. Wood adhesives. In: CHAUDHURY, M., POCIUS, A.V. (Ed.)

Adhesive science and engineering 2: surfaces, chemistry and applications. Amsterdam: Elsevier, 2002. p.1039-1103.

\section{EUROPEAN COMMITTEE FOR}

STANDARDIZATION. EN 300: Oriented Strand Boards (OSB) - definitions, classification and specifications. Bruxelas: 2006.

GOULART, S. L.; MORI, F. A.; DE ALMEIDA, N. F.; MENDES, R. F.; MENDES, L. M. Resistência ao cisalhamento de painéis compensados produzidos com adesivo à base de taninos de Stryphnodendron adstringens (barbatimão). Floresta e Ambiente, v.19, n.3, p.308-315, 2012.

IWAKIRI, S. Painéis de madeira reconstituída. Curitiba: Fundação de Pesquisas Florestais do Paraná - FUPEF, 2005, 247 p.

MALONEY, T.M. Modern particleboard \& dry process fiberboard manufacturing. San Francisco: M. Freeman, 1993. 681p.

MORI, F. A.; MORI, C. L. S. O.; MENDES, L. M.; DA SILVA, J. R. M.; DE MELO, V. M. Influência do sulfito e hidróxido de sódio na quantificação em taninos da casca de barbatimão (Stryphnodendron adstringens). Floresta e Ambiente, v. 10, n.1, p.86-92, 2003.
MOUBARIK, A.; PIZZI, A.; ALLAL, A.; CHARRIER, F.; CHARRIER, B. Quantificação de taninos das cascas de barbatimão

(Stryphnodendron adstringens). In: ENCONTRO BRASILEIRO EM MADEIRA E ESTRUTURAS DE MADEIRA, 10., 2006, São Pedro. Anais... São Pedro: [s. n.], 2006.

MOUBARIK, A.; PIZZI, A.; ALLAL, A.; CHARRIER, F.; CHARRIER, B. Cornstarch and tannin in phenol-formaldehyde resins for plywood production. Industrial Crops and Products, v.30, n.2, p.188-193, 2009.

OKINO, E.Y A., TEIXEIRA, D.E., DEL MENEZZI, C.H.S. Post-thermal treatment of Oriented Strandboard (OSB) made from cypress (Cupressus glauca Lam.). Maderas: Ciencia y tecnología, v.9, n.3, p.199-210, 2007.

PING, L.; PIZZI, A.; GUO, Z. D.; BROSSE, N. Condensed tannins extraction from grape pomace: Characterization and utilization as wood adhesives for wood particleboard.

Industrial Crops and Products, v.34, p.907-914, 2011

PIZZI, A. Recent developments in eco-efficient bio-based adhesives for wood bonding: opportunities and issues. Journal of Adhesion Science and Technology, v.20, n.8, p.829-846, 2006.

PIZZI, A. Tannin-based wood adhesives. In: PIZZI, A. Advanced wood adhesives technology. New York: M. Dekker, 1994. p.149-217.

SALARI, A.; TABARSA, T.; KHAZAEIAN, A.; SARAEIAN, A. Improving some of applied properties of oriented strand board (OSB) made from underutilized low quality paulownia (Paulownia fortunie) wood employing nano$\mathrm{SiO}^{2}$. Industrial Crops and Products, v.42, n.1, p.1-9, 2013.

SELLERS Jr, T., MILLER Jr, G.D. Laboratory manufacture of high moisture southern pine strandboard bonded with three tannin adhesive types. Forest Products Journal, v.54, n.12, p.296-301, 2004. 
TEODORO, A.S.; LELIS, R.C.A. Extração de taninos da casca de Eucallyptus pellita e avaliação de suas propriedades. Revista da Universidade Rural: Série Ciências da Vida, v.23, n.2, p.65-70, 2003.

TROSA, A.; PIZZI, A. A no-aldehyde emission hardener for tannin-based wood adhesives for exterior panels. Holz als Roh- und Werkstoff, v.59, p.266-271, 2001.

VITAL, B. R.; CARNEIRO, A. C. O.; PIMENTA, A. S.; DELLA LUCIA, R. M. Adesivos à base de taninos das cascas de duas espécies de eucalipto para produção de chapas de flocos. Revista Árvore, v.28, n.4, p.571-582, 2004. 
